\title{
Efecto del Almacenamiento de Granos de Maíz (Zea mays) sobre la Calidad del Aceite Extraído
}

Carlos Hernández, Ysamar Rodríguez, Zulay Niño y Sergio Pérez

Universidad de Carabobo, Facultad de Ingeniería, Escuela de Ingeniería Química, Avenida Bolívar 125-39, Valencia-Venezuela

(e-mail: carherna@uc.edu.ve, ysa_r@hotmail.com, znino@uc.edu.ve,sperez@uc.edu.ve)

\section{Resumen}

Se evaluó el efecto del almacenamiento de granos de maíz amarillo (Zea mays) sobre la calidad del aceite obtenido del mismo. Se determinaron los parámetros de calidad del maíz al momento de la recepción, los índices de acidez, peróxido, y el color del aceite extraído de granos almacenados en un silo de concreto por 0, 30, 60 y 90 días, utilizando los procedimientos descritos en las normas vigentes en Venezuela. Se encontró un efecto adverso significativo $(P<0.05)$ del almacenamiento sobre los índices analizados, causado por la humedad e impurezas del grano recibido, así como una deficiencia de $704 \mathrm{~m}^{3} \mathrm{~min}^{-1}$ de aire fresco suministrado al silo, lo cual no favorece un adecuado almacenaje. El grano de maíz almacenado durante 90 días produjo incrementos apreciables en el índice de acidez, índice de peróxido y color del aceite crudo obtenido.

Palabras clave: granos de maíz, almacenamiento de maíz, calidad del aceite, índice de acidez

\section{Effect of Grains of Maize (Zea mays) Storage on the Quality of the Extracted Oil}

\begin{abstract}
The effect of storage time on grains of yellow maize (Zea mays) was evaluated on the quality of the oil obtained. The quality parameters detected at the reception of the maize were: indexes of acidity, peroxide and the color of the oil extracted from grains stored in a concrete silo for 0, 30, 60 and 90 days, using the procedures described in the patterns in Venezuela. Adverse significant effects $(P<0.05)$ of the storage time on the indexes analyzed were found. These were caused by the moisture and impurities of the grains, as well as a deficiency of $704 \mathrm{~m}^{3} \mathrm{~min}^{-1}$ of fresh air fed to the silo, which affects storage. The grain of maize stored during 90 day produced increases in the index of acidity, index of peroxide and color of the extracted oil.
\end{abstract}

Keywords: grains of maize, storage of maize, oil quality, index of acidity 


\section{INTRODUCCIÓN}

La calidad del aceite de maíz comienza en el campo, ya que las condiciones de cultivo, el desarrollo del mismo y el tratamiento que recibe la semilla hasta su almacenaje, influyen su calidad. La calidad de los granos no sólo es afectada por las operaciones de secado, sino también por factores ambientales como la temperatura y la humedad durante el llenado de los mismos, por las prácticas de almacenamiento y por su transporte (INTA, 2006).

De acuerdo a Alfaro et al. (2004), en Venezuela, el maíz amarillo representa apenas 10\% de la producción nacional, el 90\% restante es proveniente en su mayoría de los Estados Unidos (USA). El maíz producido en USA se mantiene frío y húmedo durante la época de cosecha y durante los primeros seis meses de almacenaje. Según Reed (2001), almacenar maíz del USA enfrenta desafíos difíciles, ya que el proceso de exportación con tanto manejo del grano causa más rotura y polvo, por otra parte, la temperatura ambiente y la humedad relativa son frecuentemente más altas en climas tropicales como el de Venezuela que en los USA.

Los granos almacenados constituyen un agroecosistema complejo por la serie de interacciones producidas entre luz, temperatura, humedad y agentes bióticos como insectos y hongos que repercuten en la calidad del grano de maíz (Olakojo y Akinlosotu 2004; Neethirajan et al., 2007). Durante el almacenamiento ocurre el fenómeno de la respiración, causada por el propio grano, dando origen a actividades metabólicas de los seres vivos allí presentes, produciendo energía y agua, que tienden a acumularse en el propio lugar donde se generaron, formando focos de calentamiento que son los primeros indicios de un proceso deteriorativo del producto almacenado (Alabadan y Oyewo, 2005). Los granos de maíz deben almacenarse de tal forma que no se deteriore su calidad. Lo anterior puede lograrse si se controla la humedad del grano, la humedad relativa y la temperatura ambiente, ya que son determinantes para su conservación (Rosas et al., 2007).

El contenido de humedad es muy importante, ya que si es mayor a $15,5 \%$, durante su almacenamiento pueden crecer hongos e insectos y producirse pérdidas importantes (Méndez et al. 2005), debido a la producción de las toxinas y deterioración del grano (Charm, 2007). De acuerdo a Mngadi et al. (2008) y Wagacha y Muthomi (2008), la formación de micotoxinas en granos infestados de hongos, como consecuencia de la humedad y temperatura, son un potencial peligro para la salud humana y animal, con el agravante de una baja calidad del producto que se elabora.

Las grasas y los aceites pueden sufrir diferentes transformaciones y reducir el valor nutritivo del alimento, producen compuestos volátiles que imparten olores y sabores desagradables (Badui, 2006). La hidrólisis de los lípidos en los granos almacenados tiene lugar mucho más rápido que la hidrólisis de las proteínas o de los carbohidratos. Por esta razón, el contenido de ácidos grasos libres ha sido propuesto como un índice sensible del incipiente deterioro de los granos (Dhingra et al., 1998)

Narayan et al. (1988), estudiaron los cambios en la calidad de los granos de soya durante el almacenamiento y su efecto en las propiedades fisicoquímicas y sensoriales, encontrando incrementos en los ácidos grasos libres e índice de peróxido en el aceite obtenido de los granos. Por otra parte, los estudios realizados por Dhingra et al. (1998), sobre la acumulación de ergosterol y los cambios en la calidad del aceite de granos de soya almacenados e invadidos por Aspergillus ruber, demostraron un incremento en el contenido de ácidos grasos libres. Iguales resultados fueron obtenidos por Bhattacharya y Raha (2002) en su investigación sobre el deterioro de maíz, maní y soya almacenados con diferentes especies de hongos.

Otras investigaciones como las realizadas por Rehman et al. (2002), Rehman (2006) y Weinberg et al. (2008), se han avocado al estudio en el cambio nutricional del maíz y otros cereales durante el almacenamiento a diferentes condiciones de temperatura y humedad, sin embargo, ha sido poco reportado el impacto de estas condiciones sobre la calidad del aceite y en ninguna de ellas se han realizado propuestas concretas sobre el mejoramiento de las condiciones de almacenaje.

De acuerdo con lo anterior, el objetivo de esta investigación fue estudiar el efecto del almacenamiento del grano de maíz amarillo (Zea mays), en un silo de concreto, sobre la calidad del 
aceite, determinando el índice de acidez, el índice de peróxido y el color, no solo para conocer la influencia sobre estas variables, sino también proponer una solución práctica al problema.

\section{MATERIALES Y MÉTODOS}

El tipo de maíz que se utilizó fue el amarillo semiblando y dentado grado USS N², procedente de USA., recibido en el mes de junio y almacenado en un silo cilíndrico de concreto de 2.400 toneladas, dotado con un sistema de ventilación capaz de proporcionar $310 \mathrm{~m}^{3} \mathrm{~min}^{-1}$ de aire fresco. La ubicación del silo y lugar donde se efectúo la investigación fue Turmero, estado Aragua, caracterizado por un clima tropical durante todo el año, con valores promedio de humedad relativa $(71,0 \%)$ y temperatura $\left(25,8^{\circ} \mathrm{C}\right)$. Durante la recepción el grano de maíz no se fumigó, ni se usó protector de grano o inhibidor de moho. El estudio se basó en un tiempo máximo de almacenamiento de 90 días, con mediciones a intervalos de 30 días.

Para la determinación del tamaño de la muestra se utilizó la metodología indicada en la norma COVENIN 612 (1982), la cual establece que para un almacenamiento a granel, cuyo lote estuviese compuesto por 500 toneladas métricas o más de producto, se deben obtener entre 11 a 20 muestras primarias, hasta completar una muestra compuesta de $5 \mathrm{~kg}$. La toma de las muestras se realizó en el tope, centro y fondo del silo, con la ayuda de un calador cilíndrico de $4 \mathrm{~m}$ de longitud y $5 \mathrm{~cm}$ de diámetro, mezcladas posteriormente en un recipiente limpio y libre de humedad. Para la homogenización, la muestra compuesta se hizo pasar por un divisor mecánico tipo Boerner dotado de cribas metálicas y bandejas de fondo para la recepción de las muestras divididas (COVENIN 1935, 1987).

Las pruebas consistieron en determinaciones de la humedad, impurezas, granos dañados, granos quemados y granos atacados por insectos (COVENIN 1935, 1987). El procedimiento de muestreo y homogenización, señalados anteriormente, al igual que los ensayos de calidad del grano se realizaron por triplicado, calculando y reportando el promedio de las mediciones efectuadas.

La determinación de la humedad se realizó de acuerdo a la metodología descrita en la norma COVENIN 1156 (1979), la cual consistió en calcular la humedad en base a una diferencia de peso y expresarla como porcentaje. El peso de la muestra fue de $10 \mathrm{~g}$ aproximadamente, la misma se colocó en una bandeja de aluminio, previamente pesada y tarada; y se sometió a $130^{\circ} \mathrm{C}$ durante 1 hora. Finalmente se dejó enfriar la muestra en un desecador, se pesó hasta conseguir peso constante y se comparó con el peso inicial, para luego calcular el porcentaje de humedad.

De acuerdo a González (1995), las impurezas son los granos distintos a los granos de maíz (incluidos los pedazos de maíz, granos pequeños y trozos de cualquier materia que pasan a través de una criba con perforaciones circulares de $4,75 \mathrm{~mm}$ de diámetro) y cualquier material, que no siendo maíz, permanezca sobre la criba después del cribado. La determinación consistió en pesar la muestra original, cribando posteriormente los granos de maíz en porciones de $250 \mathrm{~g}$ a través de una criba de aberturas circulares de 4,75 mm de diámetro. Se pesó la materia extraña sobre la criba y la que pasó a través de ésta, expresando el resultado en porcentaje al dividir masa de las impurezas entre la masa de la muestra original (COVENIN 1935, 1987).

Los granos dañados son granos enteros o partidos que están marcadamente dañados por calor, factores climáticos, ataques de hongos, insectos, roedores y que estén germinados. Se considera un grano dañado cuando el daño es claramente visible y de tal carácter que sea reconocido como perjudicial al comercio o utilización (González, 1995). La determinación se realizó de acuerdo a la norma COVENIN 1935 (1987), la cual consistió en pesar 250 g de muestra homogenizada aproximadamente, separando a mano los granos dañados y pesándolos posteriormente, expresando el resultado en porcentaje en peso.

Los granos quemados son aquellos que han sido carbonizados total o parcialmente por calor externo. Su determinación se basó en la norma COVENIN 1935 (1987), para ello se pesaron $250 \mathrm{~g}$ de muestra homogenizada, retirando a mano los granos quemados presentes en la muestra y pesándolos posteriormente, expresando el resultado en porcentaje en peso. 
Los granos atacados por insectos, es aquel grano o pedazo del grano de maíz con presencia de insectos $u$ otras plagas dañinas en cualquiera de sus estadios biológicos (huevos, larvas, etcétera). Fue determinado según los lineamientos de la norma COVENIN 1935 (1987), la cual indica que de la totalidad de la muestra se deben separar manualmente los granos con presencia de daños por insectos, expresando el resultado en porcentaje.

Para facilitar la extracción del aceite crudo, los granos de maíz fueron secados en una estufa a una temperatura de $100^{\circ} \mathrm{C}$ durante 1 hora, reduciéndoles posteriormente el tamaño mediante el uso de un molino mecánico marca Quaker City Mill, modelo 4-E, de tiempo continuo y $86 \mathrm{rpm}$, del cual fueron obtenidos diferentes tamaños de partículas ajustando la abertura de los platos reductores. La extracción del aceite se realizó con hexano grado analítico como solvente extractor en un equipo Soxhlet, siguiendo el procedimiento descrito en la norma COVENIN 3218 (1996), el cual consistió en pesar $10 \mathrm{~g}$ de muestra que se colocaron en dedales de celulosa. El hexano se colocó en balones de $250 \mathrm{~mL}$ hasta ocupar $3 / 4$ del volumen total. Se inició el calentamiento y se dejó 5 horas el proceso de extracción. Tanto a la muestra original (0 días), como al maíz almacenado por espacio de 30, 60 y 90 días también se les aplicó la metodología antes indicada.

Las variables que se utilizaron para verificar la calidad del aceite fueron el índice de peróxido, el índice de acidez y color. Estas mediciones se efectuaron al aceite crudo obtenido del maíz en la recepción del mismo (0 días de almacenamiento) y al maíz almacenado por 30, 60 y 90 días. La toma de muestras y homogenización se realizó de acuerdo a las normas COVENIN 612 (1982) y COVENIN 1935 (1987). Los ensayos fueron realizados por triplicado, reportando el valor promedio de las mediciones y la desviación estándar correspondiente.

Se determinó el índice de peróxido en el aceite crudo, el cual se basa en la capacidad del oxígeno peroxídico de oxidar el ión yoduro del yoduro de potasio (KI) produciendo yodo, el cual se valoró con una solución de tiosulfato de sodio. Para realizar esta prueba se siguió la norma COVENIN 0508 (2001). Se pesó aproximadamente $5 \mathrm{~g}$ de aceite, el cual se diluyó en una solución ácido acéticocloroformo y se hizo reaccionar con yoduro de potasio por un minuto, luego se le adicionó gran cantidad de agua y al observar cambio de color, se tituló con tiosulfato de sodio, la prueba también fue realizada sin muestra para obtener el blanco respectivo.

El índice de acidez se determinó de acuerdo a la norma COVENIN 0325 (2001) y se basó en la reacción de neutralización de los ácidos grasos con una base. Para ello se preparó una solución alcohólica del aceite, agregando $75 \mathrm{~mL}$ de alcohol etílico al 95\% y titulando posteriormente con hidróxido de sodio $0,1 \mathrm{~N}$. Se usó $4 \mathrm{~mL}$ de fenolftaleína como indicador y se expresó el resultado como porcentaje de ácido oleico.

El color del aceite se determinó a partir de la norma COVENIN 1191 (1996), aplicando un método colorimétrico usando un equipo Lovibond. El método consistió en combinar filtros de color amarillo, rojo y azul que más se asemejaban a la muestra problema. En una cubeta limpia y seca de 2,5 pulgadas, se colocó la muestra de aceite a temperatura ambiente. Luego se encendió el tintómetro Lovibond y se colocó la cubeta dentro del mismo, de tal forma que el haz de luz pasase a través de éste. Se cerró la tapa del tintómetro y se introdujeron los vidrios Lovibond en el campo de comparación, observando a través de los tubos de visión los colores de la muestra.

Para determinar el flujo de aire requerido para mantener frío el grano húmedo, fue necesario calcular el volumen del silo aplicando la ecuación 1 (González, 1995).

$\mathrm{V}_{\mathrm{s}}=\frac{\mathrm{CsXF}_{\mathrm{C} 1}}{\mathrm{DMAiZXF}_{\mathrm{C} 2}}$

donde,

$\mathrm{V}_{\mathrm{S}}$ : volumen total del silo, $\mathrm{m}^{3}$

$\mathrm{C}_{\mathrm{s}}$ : capacidad total del silo, $2400 \mathrm{t}$.

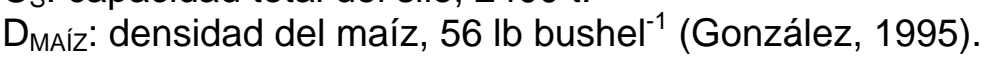


$\mathrm{F}_{\mathrm{C} 1}$ : factor de conversión de unidades $1,\left(2200 \mathrm{lb} \mathrm{t}^{-1}\right)$.

$\mathrm{F}_{\mathrm{C} 2}$ : factor de conversión de unidades $2,\left(27,78 \mathrm{~m}^{3}\right.$ bushel $\left.^{-1}\right)$.

El flujo de aire requerido se determinó con la ecuación 2 (González, 1995). De acuerdo a Reed (2001), la tasa de aire recomendada para climas tropicales es de $0,3\left(\mathrm{~m}^{3} \mathrm{~min}^{-1}\right) \mathrm{m}^{-3}$.

$F_{\text {AIRE }}=V_{S X T}$ AIRE

donde,

$\mathrm{F}_{\mathrm{AIRE}}$ : flujo de aire requerido en el silo de almacenamiento, $\mathrm{m}^{3} \mathrm{~min}^{-1}$

$\mathrm{T}_{\mathrm{AIRE}}$ : tasa de aire recomendada para climas tropicales, $\left(\mathrm{m}^{3} \mathrm{~min}^{-1}\right) \mathrm{m}^{-3}$

\section{RESULTADOS Y DISCUSIÓN}

\section{Evaluación de la calidad del grano de maíz}

En la tabla 1, se observa que la humedad se encuentra por encima de lo que recomienda la norma COVENIN 1935 (1987) para un maíz que tendrá utilidad industrial, pero ligeramente menor que el valor crítico comprendido entre $14,5 \%$ y $15 \%$, recomendado por Molteberg et al. (1995). Este valor pudiese indicar el desempeño que tendrá el grano de maíz durante el almacenamiento, debido a que el grano respira, es decir, la semilla absorbe oxígeno del aire y consume carbohidratos de su estructura, liberando calor. La respiración es más rápida cuando el grano está caliente y húmedo (situación natural del sitio de estudio) que cuando está fresco y seco.

Este análisis coincide con los estudios realizados por Jiménez et al. (2004), el cual estableció que el grano almacenado con humedad mayor a 15\% respira más rápido que el seco, produciendo más calor y creando condiciones favorables para el crecimiento de hongos y para el ataque de insectos, debido a la formación de puntos calientes. Además si se satura el aire circundante, se presenta condensación de la humedad, favoreciendo el crecimiento de microorganismos.

Tabla 1: Calidad del grano de maíz al momento de la recepción

\begin{tabular}{|c|c|c|}
\hline Análisis & Resultado & $\begin{array}{c}\text { Especificación según norma } \\
\text { COVENIN 1935 (1987) }\end{array}$ \\
\hline Humedad (\%) & 14,82 & 12 \\
\hline Impurezas (\%) & 4,82 & 2 \\
\hline Granos dañados (\%) & 4,14 & 8 \\
\hline Granos quemados (\%) & 0,02 & 0 \\
\hline Granos atacados por insectos (\%) & 0,00 & 0 \\
\hline
\end{tabular}

Por otra parte, cuando el contenido de humedad de los granos almacenados aumenta, también lo hace el espacio de aire entre los mismos, lo que contribuye a una mayor resistencia del flujo de aire a través del grano. En este sentido, Jiménez et al. (2004) demostraron que la migración de la humedad del grano prevalece, por lo cual se requiere de mayor tiempo para que el contenido de agua en el grano comience a estabilizarse, por efecto del movimiento de aire intersticial.

El valor de impurezas encontrado resultó apreciablemente superior al recomendado por la norma COVENIN 1935 (1987), pudiéndose inferir a partir de este que los numerosos movimientos a los que los gráneles se ven sometidos (desde los campos de cultivos hasta su destino final), dañan físicamente los granos y hacen que estos pierdan su protección natural contra los hongos de almacenamiento. Producto de estos movimientos, también se generan granos quebrados y polvo, cuyo porcentaje crece con cada manipulación, lo que genera durante el llenado del silo, el fenómeno de segregación de los componentes del granel y la consiguiente acumulación de los granos quebrados y del polvo alrededor del lugar donde golpea el chorro del grano (generalmente en el centro del silo). Se forma así una zona en el granel, en la cual se restringe grandemente la 
circulación natural del aire, y también la circulación forzada del aire (aireación), dando origen a un posible foco de calentamiento en dicha zona.

La acumulación de finos en la columna central del silo promueve el calentamiento en esa zona. Esto se da porque el polvo y grano quebrado son más fácilmente invadidos por hongos, como también por el hecho de que la aireación, al ser restringida en esa sección del silo, no remueve eficientemente el calor que se genera y éste llega a acumularse. Situación similar fue encontrada por Guehi et al. (2008), observando un elevado y creciente contenido de ácidos grasos libres en granos de cacao defectuosos, atribuible probablemente a la actividad de la microflora, asociada con la calidad inicial y la pérdida de la integridad física de los granos.

Por otra parte, los granos de la capa superior del silo permanecen húmedos, lo que conforma una zona de riesgo, con posibles pérdidas de materia seca y calidad del grano. Esta situación es aun más crítica en regiones de climas cálidos como en Venezuela, donde las temperaturas son elevadas, lo cual aumenta la actividad biológica en la capa superior del silo (mayor respiración del grano, hongos e insectos).

\section{Evaluación de la calidad del aceite crudo de maíz}

En la tabla 2, se muestra la evaluación de la calidad del aceite crudo de maíz para diferentes períodos de almacenamiento de los granos. Del análisis estadístico de los datos $(P<0,05)$, se observan incrementos significativos en los índices de acidez y peróxido, así en el color tanto rojo como amarillo, para cada período de almacenamiento.

Tabla 2: Evaluación de la calidad del aceite de maíz

\begin{tabular}{|c|c|c|c|c|}
\hline \multirow{2}{*}{ Variable de calidad } & \multicolumn{3}{|c|}{ Período de almacenamiento del maíz (días) } \\
\cline { 2 - 5 } & 0 & 30 & 60 & 90 \\
\hline Índice de acidez (\%) & $1,90 \pm 0,09$ & $2,12 \pm 0,08$ & $2,40 \pm 0,08$ & $2,67 \pm 0,07$ \\
\hline Índice de peróxido (mileq. $\mathrm{O}_{2} \mathrm{~kg}^{-1}$ aceite) & $3,63 \pm 0,05$ & $4,53 \pm 0,08$ & $6,20 \pm 0,06$ & $6,63 \pm 0,08$ \\
\hline Color & & & & \\
Rojo & $4,8 \pm 0,3$ & $5,9 \pm 0,5$ & $7,0 \pm 0,2$ & $7,8 \pm 0,3$ \\
Amarillo & $22,1 \pm 0,1$ & $22,9 \pm 0,3$ & $24,2 \pm 0,4$ & $25,7 \pm 0,6$ \\
\hline
\end{tabular}

El índice de acidez del aceite y de peróxido obtenido con los granos de maíz almacenados durante 90 días representa un $40,5 \%$ y $82,6 \%$ de incrementos con respecto al medido en el momento de la recepción (0 días de almacenamiento). Esto es debido a que las grasas en los granos son fácilmente descompuestas por las lipasas en ácidos grasos libres y glicerol durante el almacenaje, particularmente cuando la temperatura y la humedad son altas (Badui, 2006). El deterioro es altamente acelerado por la presencia de hongos debido a la alta actividad lipolitica de estos organismos, del cual se presume la existencia y desarrollo durante el lapso de almacenamiento estudiado.

En relación a lo anterior es importante señalar que el aceite de maíz es rico en ácidos grasos insaturados, el desarrollo de la rancidez de estos aceites es producto de reacciones de deterioro de los lípidos presentes en el grano. La mayoría de los lípidos en los granos están concentrados en las capas más externas de estos. Las lipasas presentes, las cuales son de origen endógeno o por la acción microbial, es decir, producidas por insectos y hongos, entre otros, se ponen en contacto con los lípidos del grano de maíz, originando ácidos grasos libres que posteriormente por la actuación de la lipoxigenasa, autoxidación y fotoxidación producen hidroperóxidos, dando origen a aldehídos, cetonas, dimeros, polímeros, entre otros compuestos no deseados (Lehtinen y Laakso, 2004), lo que se traduce en incremento del índice de peróxido tal como se muestra en la tabla 2.

La velocidad de formación de los ácidos grasos libres depende del grado de humedad, acción microbial, temperatura de la masa del grano y tiempo de almacenamiento (Lehtinen y Laakso, 2004). El contenido de humedad crítico para almacenamiento se encuentra entre 14,5 y $15 \%$, arriba de ese 
nivel las lipasas incrementan su actividad produciendo más ácidos grasos libres y oxidación lipídica (Molteberg et al. 1995).

Romero et al. (2008), realizaron un estudio sobre los factores químicos y bioquímicos que promueven el aumento de acidez en el aceite obtenido de granos de maíz durante las etapas de almacenamiento en silos, desgerminación y peletizado de una empresa aceitera, encontrando actividad lipolítica en muestras no sometidas a tratamientos físicos de proceso, pudiéndose indicar a partir de este hallazgo, que efectivamente la acción enzimática y microbial contribuyen al deterioro progresivo del maíz durante su almacenamiento, lo que conlleva a un efecto negativo en la calidad del aceite obtenido.

En relación al color rojo y amarillo presente en el aceite de maíz, se le atribuye a la presencia de pigmentos carotenoides cuya estructura básica es un tetraterpeno de 40 átomos de carbono simétrico y lineal, que presenta inestabilidad química asociada a su estructura insaturada que los hace susceptibles a reacciones de oxidación y degradación acelerada por los radicales libres que se forman en la oxidación lipídica (Belén et al., 2004; Moreno et al., 2003). En este sentido, se observa el aumento en las mediciones del color rojo y amarillo en $62,5 \%$ y $16,3 \%$ respectivamente, por las razones antes señaladas.

El resultado obtenido del efecto del almacenamiento de los granos de maíz sobre la calidad del aceite obtenido a partir de estos, coincide con los diferentes estudios efectuados en el tema. Narayan et al. (1988), encontraron incrementos en los ácidos grasos libres e índice de peróxido en el aceite obtenido de los granos de soya durante el almacenamiento de los mismos. Aun cuando esta investigación no determinó el color del aceite, encontró cambios en la coloración de los granos, pasando de amarillo crema a marrón, lo que evidencia la degradación del pigmento carotenoide, tal como ocurre en los granos de maíz analizados.

Igualmente se obtuvieron resultados similares a los estudios realizados por Dhingra et al. (1998) sobre los cambios en la calidad del aceite de granos de soya almacenados e invadidos por Aspergillus ruber, en los que demostraron un incremento en el contenido de ácidos grasos libres, producto de la acción de las lipasas. Iguales resultados fueron obtenidos por Bhattacharya y Raha (2002) en su investigación sobre el deterioro de maíz, maní y soya almacenados con diferentes especies de hongos.

\section{Flujo de aire requerido en el silo de almacenamiento de los granos de maíz}

De acuerdo al diseño del silo donde se almacenaron los granos de maíz, el flujo de aire fresco máximo, capaz de proporcionar el sistema de ventilación fue de $310 \mathrm{~m}^{3} \mathrm{~min}^{-1}$, sin embargo al calcular los requerimientos reales de flujo de aire para las condiciones del silo, utilizando para ello las ecuaciones 1 y 2 mostradas en la sección de metodología, resultó que el caudal de aire debiese ser de $1.014 \mathrm{~m}^{3} \mathrm{~min}^{-1}$, esto representa un deficiencia de $704 \mathrm{~m}^{3} \mathrm{~min}^{-1}$ de aire fresco que contribuye de forma apreciable al calentamiento del grano de maíz en el silo, con la consecuencia del deterioro progresivo del mismo durante su almacenamiento (Reed, 2006), afectando la calidad del aceite obtenido como se observa en la tabla 2.

En este mismo orden de ideas es importante señalar los trabajos realizados por Volenik et al. (2007) y Alabadan (2006), el cual establecieron que la temperatura es el factor más importante que afecta la calidad del grano durante su almacenamiento, como consecuencia del crecimiento de hongos e insectos. El calor metabólico liberado por la infestación de insectos en el grano es la principal causa del cambio de temperatura durante el almacenamiento, cuyo mecanismo de transferencia se realiza por conducción, produciéndose un gradiente de temperatura por convección. La temperatura y la humedad actúan como catalizadores de los procesos metabólicos, aumentando la tasa de respiración de los granos y las pérdidas de materia seca. A mayor temperatura y humedad, mayor riesgo en la conservación de los granos, y a su vez un grano que no esta sano y limpio es más propenso a ser atacado por hongos e insectos (Newman, 2003), cuya acción microbial origina la producción de lipasa que hidroliza los triglicéridos y forma ácidos grasos libres y peróxidos tal como se observa en el incremento significativo $(P<0,05)$ en los índices de acidez, peróxido y color. 
El trabajo de Molteberg et al. (1995) sobre el efecto del almacenaje y calor de procesamiento sobre el contenido y composición de ácidos grasos libres en avena, corrobora lo indicado anteriormente, ya que en dicho estudio se determinó un incremento en el contenido de ácidos grasos libres durante el almacenamiento, atribuido a la actividad de la lipasa. Igualmente se encontró que la estabilidad del almacenaje se afectó por la temperatura, ya que una disminución de esta de 20 a $15^{\circ} \mathrm{C}$ disminuía el riesgo de infestación y resultaba en una disminución de la degradación lipídica.

Con respecto al contenido de impurezas mostrada en la tabla 1, resultó un factor de mucha importancia, ya que la presencia de éstos aumenta la resistencia al flujo de aire al disminuir el espacio libre entre los granos, ya que el material más pequeño y pesado se ubica en el centro del granel, obstruyendo la normal salida de aire de los conductos de aireación, que como se indicó se encuentra considerablemente por debajo de lo requerido para la capacidad del silo, representando una condición no favorable para el almacenamiento del maíz.

\section{Propuesta de solución al problema}

En relación a la deficiencia de aire fresco suministrado al silo, se plantea como una posible alternativa de solución, la modificación del sistema de aireación, basado en la sustitución del ventilador por otro capaz de suministrar la cantidad de aire necesario, el cual es de $1.014 \mathrm{~m}^{3} \mathrm{~min}^{-1}$. La técnica de aireación se basa en utilizar al aire como fluido que intercambia energía, en forma de calor o de agua, con los granos, logrando el enfriamiento de los mismos y disminuyendo la actividad metabólica, controlando de manera indirecta a través de la disminución de la temperatura y humedad, el desarrollo de hongos e insectos (Newman, 2003). Estudios realizados por Flinn et al. (2004), reafirman que la aireación adecuada de silos, es una alternativa efectiva como mecanismo de control en la formación y crecimiento de insectos.

\section{CONCLUSIONES}

Se evidenció un valor de impurezas por encima de lo recomendado por la normativa nacional vigente para un maíz de utilidad industrial.

El grano de maíz almacenado durante 90 días produjo incrementos apreciables en el índice de acidez, índice de peróxido y color del aceite crudo obtenido.

El flujo de aire suministrado al silo de almacenamiento de maíz se encontró por debajo de los requerimientos reales, con una deficiencia de $704 \mathrm{~m}^{3} \mathrm{~min}^{-1}$.

Una solución al problema, sería la instalación de un nuevo ventilador en el sistema de aireación del silo, capaz de suministrar un caudal de aire fresco de $1.014 \mathrm{~m}^{3} \mathrm{~min}^{-1}$.

\section{REFERENCIAS}

Alabadan, B.A.; Temperature Changes in Bulk Stored Maize, Assumption University Journal of Technology: 9(3), 187-192 (2006).

Alabadan, B.A. y O.A. Oyewo; Temperature Variations within Wooden and Metal Grain Silos in the Tropics During Storage of Maize (Zea mays), Leonardo Journal of Sciences: 6(1), 59-67 (2005).

Alfaro, Y. y otros cinco autores; El Maíz Amarillo para la Molienda Húmeda, Revista Digital CENIAP HOY, ISSN: 1690-4117 (en línea) 6(3), 2004. http://www.ceniap.gov.ve/ceniaphoy/articulos/n6/arti/ alfaro_y/arti/alfaro_y.htm. Acceso: 25 de marzo del 2008.

Badui, S.; Química de los Alimentos, 4ª edición, pp. 68-180, Longman, México (2006).

Belén, D., M. Moreno, R. Alemán y F. Álvarez; Efecto de la Temperatura de Secado sobre la Degradación de Carotenoides en Frutos de Coroba (Jessenia polycarpa Karst), Ciencia y Tecnología Alimentaria: 4(3), 206-210 (2004). 
Bhattacharya, K. y S. Raha; Deteriorative Changes of Maize, Groundnut and Soybean Seeds by fungi in Storage, Mycopathologia: 155(3), 135-141 (2002).

Charm, S.; Food Engineering Applied to Accommodate Food Regulations, Quality and Testing, Alimentos Ciencia e Ingeniería: 16(1), 5-8 (2007).

COVENIN 0325; Norma Venezolana sobre Aceites y Grasas Vegetales. Determinación de la Acidez, 1-6, Caracas, Venezuela (2001).

COVENIN 0508; Norma Venezolana sobre Aceites y Grasas Vegetales. Determinación del Índice de Peróxidos, 1-6, Caracas, Venezuela (2001).

COVENIN 612; Norma Venezolana sobre Cereales - Leguminosas - Oleaginosas y Productos Derivados. Muestreo, 1-24, Caracas, Venezuela (1982).

COVENIN 1156; Norma Venezolana sobre Alimentos para Animales. Determinación de Humedad, 110, Caracas, Venezuela (1979).

COVENIN 1191; Norma Venezolana sobre Aceites y Grasas Vegetales. Determinación del Color, 1-4, Caracas, Venezuela (1996).

COVENIN 1935; Norma Venezolana sobre Maíz para Uso Industrial, 1-14, Caracas, Venezuela (1987).

COVENIN 3218; Norma Venezolana sobre Alimentos. Determinación de la Grasa Libre, 1-7, Caracas, Venezuela (1996).

Dhingra, O.D., J. Gulab e I. Napoleao; Ergosterol Accumulation and Oil Quality Changes in Stored Soybean Invaded by Aspergillus rubber (A. glaucus group), Mycopathologia: 143(2), 85-91 (1998).

Flinn, P.W., B. Subramanyam y F.H. Arthur; Comparison of Aeration and Spinosad for Suppressing Insects in Stored Wheat, Journal of Economic Entomology: 97(4), 1465-1473 (2004).

González, U.; El Maíz y su Conservación, 1ª edición, 14-320, Trillas, Distrito Federal, México (1995).

Guehi, T. y otros seis autores; Impact of Cocoa Processing Technologies in Free Fatty Acids Formation in Stored Raw Cocoa Beans, African Journal of Agricultural Research: 3(3), 174-179 (2008).

INTA, Instituto Nacional de Tecnología Agropecuaria; Calidad del Grano de Maíz (2006), (en línea). http://www.produccionbovina.com/informacion_tecnica/suplementacion/80-grano_maiz.pdf. Acceso: 18 de mayo (2008).

Jiménez, H., J.L. Navarrete y E. Botello; Estudio Numérico de la Convección Natural de Calor y Masa 2-D en Granos Almacenados en Silos Cilíndricos, Agrociencia: 38(2), 325-342 (2004).

Lehtinen, P. y S. Laakso; Role of Lipid in Quality of Oat Products, Agricultural and Food Science: 13(1), 88-99 (2004).

Méndez, G. y otros cinco autores; Composición Química y Caracterización Calorimétrica de Híbridos y Variedades de Maíz Cultivadas en México, Agrociencia: 39(3), 267-274 (2005).

Mngadi, P., R. Goviden y B. Odhav; Co-occurring Mycotoxins in Animal Feeds, African Journal of Biotechnology: 7(13), 2239-2243 (2008).

Molteberg, E.L., G. Vogt, A. Nilsson y W. Frolich; Effects of Storage and Heat Processing on the Content and Composition of Free Fatty Acids in Oats, Cereal Chemistry: 72(1), 88-93 (1995). 
Moreno, M.J., D. Belén y V. Torrez; Degradación Cinética de Carotenoides Obtenidos de Frutos de Carica papaya L., Revista de la Facultad de Agronomía de la Universidad del Zulia: 20, 232-237 (2003).

Narayan, R., G.S. Chauhan y N.S. Verma; Changes in the Quality of Soybean During Storage Part 1Effect of Storage on Some Physicochemical Properties of Soybean, Food Chemistry: 27(1), 13-23 (1988).

Neethirajan, S., C. Karunakaran, D. Jayas y N. White; Detection Techniques for Stored - Product Insects in Grain, Food Control: 18(2), 157-162 (2007).

Newman, C.; Grain Storage: Maintaining Grain Quality, Farmnote № FN 65, 1-4, Government of Australia, Department of Agriculture, Australia (2003).

Olakojo, S.A. y T.A. Akinlosotu; Comparative Study of Storage Methods of Maize Grains in South Western Nigeria, African Journal of Biotechnology: 3(7), 362-365 (2004).

Reed, C.; Managing Stored Grain to Preserve Quality and Value, $1^{\text {a }}$ edición, 50-215, AACC International, St. Paul, MN (2006).

Reed, C.; U.S. Corn: Storage in Tropical Climates, U.S. Grains Council, 1-52, U.S.A, Washington D.C. (2001).

Rehman, Z.; Storage Effects on Nutritional Quality of Commonly Consumed Cereals, Food Chemistry: 95(1), 53-57 (2006)

Rehman, Z., F. Habib y S.I. Zafar; Nutritional Changes in Maize (Zea mays) During Storage at Three Temperatures, Food Chemistry: 77(2), 197-201 (2002).

Romero, D., F. Bolívar y N. Salinas; Estudio de los Posibles Eventos Químicos y Bioquímicos que Inciden en el Incremento de la Acidez en el Proceso de Extracción de Aceite de Maíz, Grasas y Aceites: 59(3), 282-287 (2008).

Rosas, I. y otros cuatro autores; Calidad Física y Fisiológica de Semilla de Maíz Criollo Almacenada en Silo Metálico y con Métodos Tradicionales en Oaxaca, México, Fitotecnia Mexicana: 30(1), 69-78 (2007).

Volenik, M. y otros cinco autores; Influence of Relative Humidity and Temperature on the Changes in Grain Moisture in Stored Soybean and Maize, Agriculturae Conspectus Scientificus: 72(3), 215-219 (2007).

Wagacha, J.M. y J.W. Muthomi; Mycotoxin Problem in Africa: Current Status, Implications to Food Safety and Health and Possible Management Strategies, International Journal of Food Microbiology: 124(1), 1-12 (2008).

Weinberg, Z.G. y otros cinco autores; The Effect of Moisture Level on High - Moisture Maize (Zea mays L.) Under Hermetic Storage Conditions - in Vitro Studies, Stored Products Research: 44(2), 136-144 (2008). 